\title{
Aqueous phase synthesis of bridgehead azaheterocycles in the presence of $\beta$-cyclodextrin
}

\author{
Shailesh Kumar and Devi Prasad Sahu* \\ Medicinal and Process Chemistry Division, Central Drug Research Institute, Chattar Manzil \\ Palace, Lucknow 226001, UP, India \\ E-mail:ㅁsahuin@yahoo.com, dp_sahu@cdri.res.in
}

\begin{abstract}
A simple and expeditious aqueous phase reaction of various $\alpha$-bromoketones with 2aminopyridine, 2-aminopyrimidine, 2-aminopyrazine, and their derivatives, and 2aminobezothiazole in the presence of $\beta$-cyclodextrin has been demonstrated to furnish bridgehead azaheterocycles 5a-j, 7a-e, 9a-d, 12a, 12b and benzo[ $d]$ imidazo[2,1- $b]$ thiazoles 11ac respectively, in good to excellent yields.
\end{abstract}

Keywords: Heterocycles, $\beta$-cyclodextrin, and azaindolizines, green chemistry

\section{Introduction}

Imidazo[1,2-a]pyridine 1, imidazo[1,2- $a$ ]pyrimidine 2 and imidazo[1,2-a]pyrazine 3 (Figure 1), are important structural motifs found in numerous natural and synthetic bioactive molecules, and have received significant attention from the pharmaceutical industry owing to their interesting biological activities displayed over a broad range of therapeutic classes. The members of these ring systems have several valuable biological properties and are used as hypotensive, ${ }^{1}$ antiulcer ${ }^{2}$ or anxyolitic agents ${ }^{3}$ in addition to bradycardic, ${ }^{4}$ antiasthmatic, ${ }^{5}$ antimicrobial, ${ }^{6}$ cytoprotective, ${ }^{7}$ calcium channel blocking agents ${ }^{8}$ and as HIF- $1 \alpha$ prolyl hydroxylase inhibitors. ${ }^{9}$ Imidazo[1,2a]pyridines are also an important pharmocophore in a number of drug formulations currently available in the market. ${ }^{10}$ Apart from the above, imidazo[1,2- $a$ ]pyridinium salts are also used to prepare styryl dyes. ${ }^{11}$<smiles>c1ccn2ccnc2c1</smiles>

1<smiles>c1cnc2nccn2c1</smiles>

2<smiles>c1cn2ccnc2cn1</smiles>

3

Figure 1. Some bioactive azaindolizine ring systems. 
An increasing amount of published literature describes new synthetic routes for the preparation of azaindolizines. These methods include either solution phase ${ }^{12}$ or solid phase, ${ }^{13}$ coupling reaction of $\alpha$-haloketones or equivalents with corresponding heteroaromatic amidines, or through multicomponent reaction by microwave-assisted reactions ${ }^{14}$ and zinc chloride catalyzed reactions. ${ }^{15}$ In spite of the existence of numerous methods ${ }^{12,16}$ for the synthesis of fused imidazoles, these methods are associated with one or more of the following drawbacks: (i) long reaction times, (ii) unsatisfactory yields, and (iii) the use of expensive and hazardous reagents. Therefore, it seems highly desirable to find a one step and inexpensive protocol for the synthesis of azaindolizines systems for their experimental simplicity and effectiveness.

Water plays an essential role in life processes, and in the development of environmentally benign and clean synthetic procedure, however its use as a solvent has been limited in organic synthesis. Despite the fact that it is the cheapest, safest and most non-toxic solvent in the world, its presence is generally avoided through the dehydrative drying of substrates and solvents. The use of water as a medium for organic reactions is therefore one of the latest challenge for modern organic chemists. A number of reactions in water have been previously reported, and the supramolecular catalysis appeared to the best method for some of the reactions. ${ }^{17}$

Cyclodextrins are cavity based cyclic oligosaccharides that catalyze chemical reactions on the basis of supramolecular catalysis. These molecules mimic the enzyme models in the formation of reversible host-guest complex by non-covalent interaction. Cyclodextrins consist of hydrophobic cavities, which selectively bind the hydrophobic portion of one of the reacting substrate and thereby catalyzing the reaction. Complexation depends on the size, shape and hydrophobicity of the guest molecule.

With in this context we have developed a biomimetic and green chemistry approach to synthesize fused imidazoles under aqueous condition through the supramolecular catalysis mediated by $\beta$-cyclodextrins. Herein, we described a simple and convenient supramolecular synthesis of some azaindolizines $\mathbf{5 a - j}$, 7a-e, 9a-d, 12a, 12b and benzo[ $d]$ imidazo[2,1- $b]$ thiazoles 11a-c, and the present method seems to overcome all of the above mentioned drawbacks. To best of our knowledge, there has been no report on cyclodextrin-mediated synthesis of bridgehead azaheterocycles.

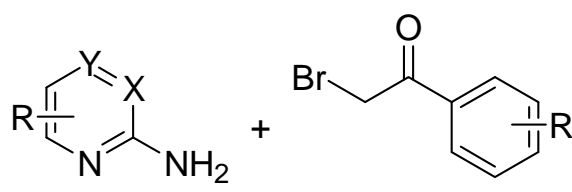

$4,6,8$

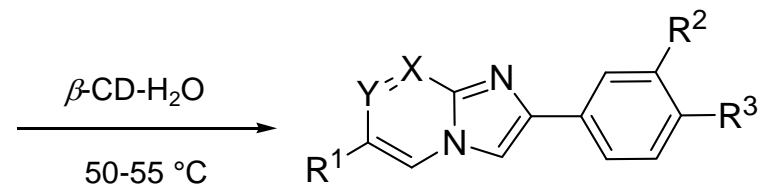

5a-j, 7a-e, 9a-d

\section{Scheme 1}




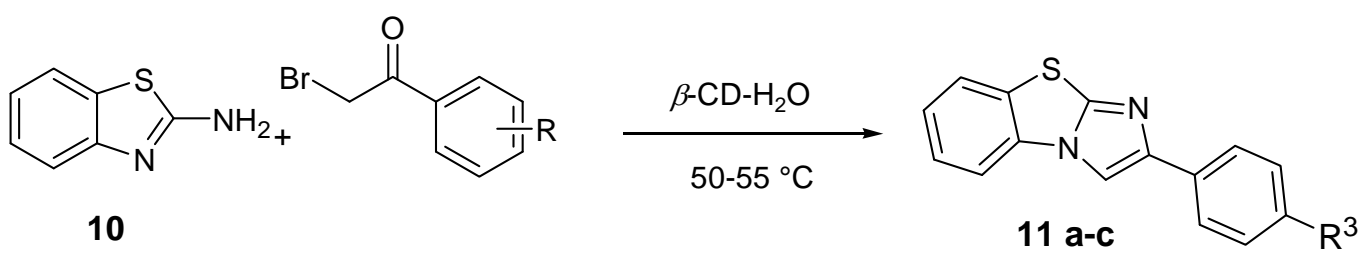

\section{Scheme 2}

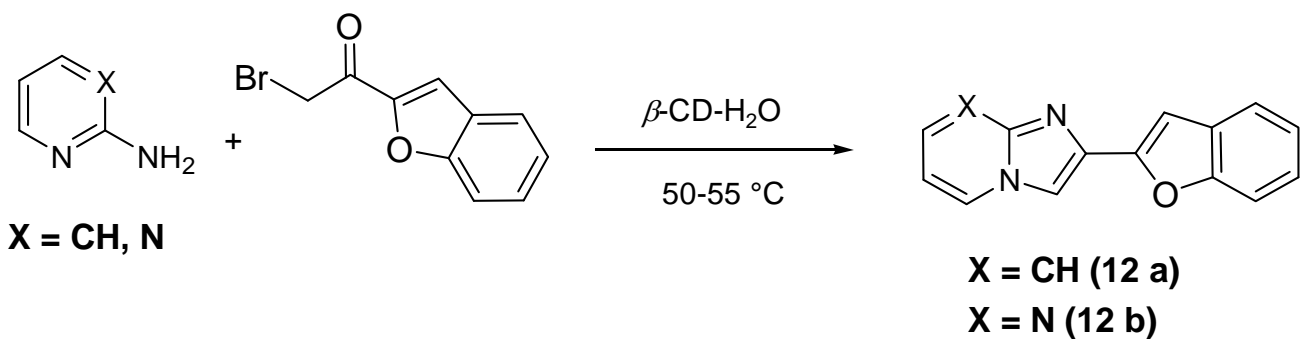

Scheme 3

\section{Results and Discussion}

To establish the generality and scope of the method given in this report, a number of azaindolizines (with two nitrogen atoms) and benzo[d]imidazo[2,1-b]thiazole derivatives have been synthesized, and the results are shown in Table 1. All the reactions were carried out with one equivalent of each reactant in the aqueous solution of one equivalent of $\beta$-cyclodextrin. The reactions were carried out by dissolving $\beta$-cyclodextrin in water warmed to $50-55{ }^{\circ} \mathrm{C}$. To resulting clear solution, phenacyl bromides were added. A milky suspension of $\beta$-CD-phenacyl bromide complex was formed, to which the amino compounds were added and stirred. The progress of reactions was monitored by TLC, and an appearance of blue fluorescent at Rf. of 0.10.4 in $10 \%$ methanol/chloroform indicated the formation of product. The reaction of various phenacyl bromides with 2-aminopyridines 4 (entry 1, Table 1), furnished the azaindolizines 5a-j. Similarly, the reaction of 2-aminopyrimidines 6 (entry 2) and 2-aminopyrazines 8 (entry 3) furnished the compounds 7a-e and 9a-d respectively. By adopting similar synthetic protocols (Scheme 2 and 3), benzo[d]imidazo[2,1-b] thiazoles 11a-c (entry 4) and azaindolizines 12a-b (entry 5) were also synthesized in good to excellent yield. The method describes an easy and convenient route to furnish a number of azaheterocycles. The superiority of this procedure over existing protocol could be established while comparing the results obtained with few methods employed previously for the synthesis of 2-arylimidazo[1,2-a] pyridines, 2-arylimidazo[1,2a]pyrimidines, as these methods ${ }^{12,18}$ needed refluxing in organic solvents for 6 hours. The formation of 2-substituted benzo[ $d]$ imidazo[2,1-b]thiazole ${ }^{20}$ needed 8 hours refluxing in dry ethanol, but the method given in this report is quite simple and the products were formed within a few minutes in very good yields. Under the similar reaction 
Table 1. Synthesis of bridgehead azaheterocycles in the presence of $\beta$-cyclodextrin

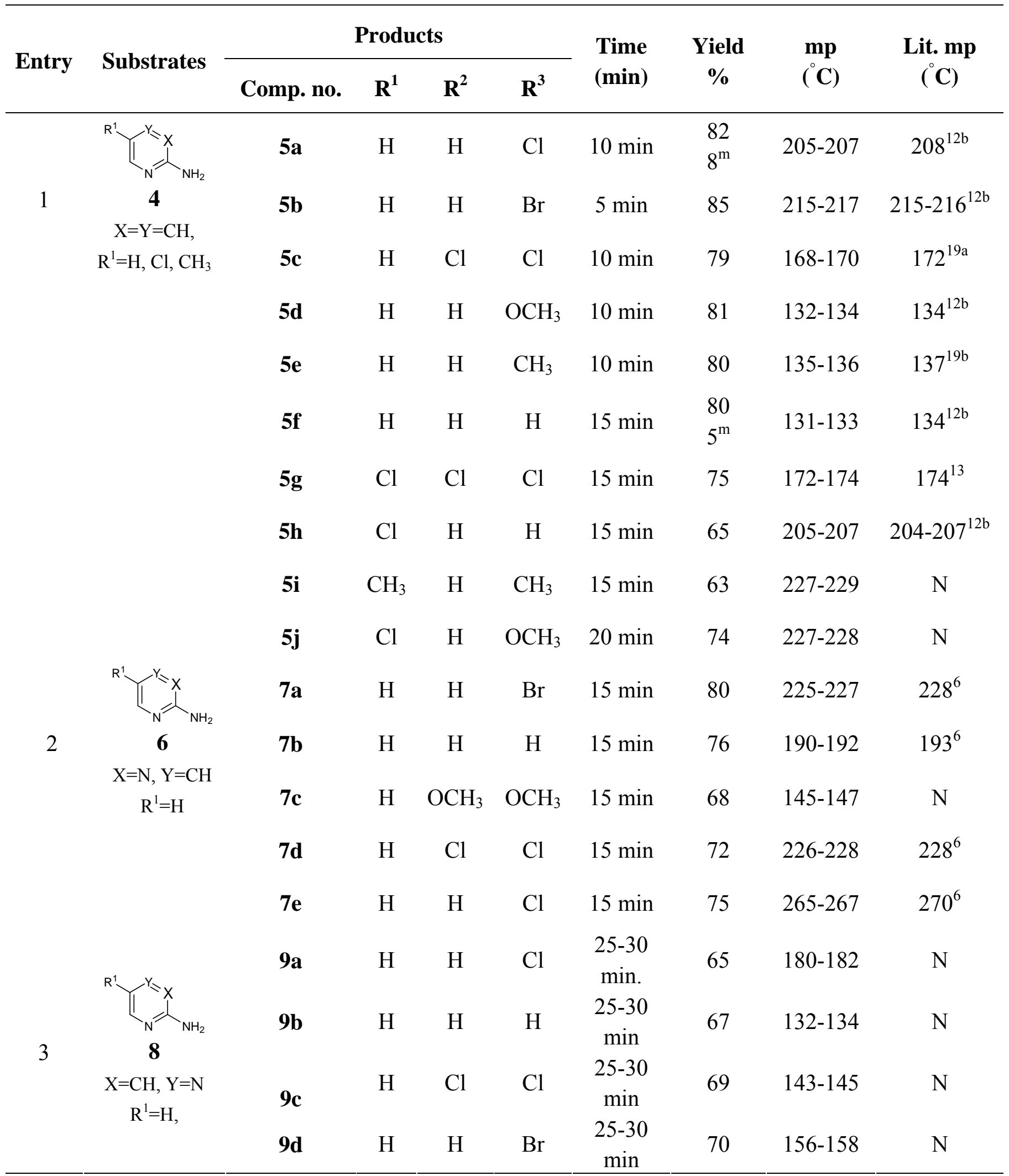


Table 1. Continued

\begin{tabular}{|c|c|c|c|c|c|c|c|c|c|}
\hline \multirow{2}{*}{ Entry } & \multirow{2}{*}{ Substrates } & \multicolumn{4}{|c|}{ Products } & \multirow{2}{*}{$\begin{array}{l}\text { Time } \\
\text { (min) }\end{array}$} & \multirow{2}{*}{$\begin{array}{c}\text { Yield } \\
\%\end{array}$} & \multirow{2}{*}{$\begin{array}{l}\text { mp } \\
\text { ( } \mathrm{C})\end{array}$} & \multirow{2}{*}{$\begin{array}{c}\text { Lit. mp } \\
\text { ( C) }\end{array}$} \\
\hline & & Comp. no. & $\mathbf{R}^{1}$ & $\mathbf{R}^{2}$ & $\mathbf{R}^{3}$ & & & & \\
\hline \multirow{3}{*}{4} & & 11a & - & $\mathrm{H}$ & $\mathrm{H}$ & $20 \mathrm{~min}$ & $\begin{array}{l}70 \\
5^{\mathrm{m}}\end{array}$ & $102-104$ & $106^{20}$ \\
\hline & 10 & $11 b$ & - & $\mathrm{H}$ & $\mathrm{Cl}$ & $20 \mathrm{~min}$ & $\begin{array}{c}70 \\
10^{\mathrm{m}}\end{array}$ & $157-159$ & $160^{20}$ \\
\hline & & 11c & & $\mathrm{H}$ & $\mathrm{OCH}_{3}$ & $20 \mathrm{~min}$ & 69 & $175-177$ & $181^{20}$ \\
\hline \multirow{2}{*}{5} & & $12 a$ & - & - & - & $5 \mathrm{~min}$ & 75 & 197-199 & $\mathrm{N}$ \\
\hline & & $12 b$ & - & - & - & $15 \mathrm{~min}$ & 74 & $174-176$ & $\mathrm{~N}$ \\
\hline
\end{tabular}

${ }^{\mathrm{m}}:$ in absence of $\beta$-cyclodextrin.

$\mathrm{N}$ : Lit. is not available.Conditions, in the absence of $\beta$-cyclodextrin only $5-10 \%$ products could be isolated.

Cyclodextrin by supramolecular interaction effects solubilization and activation of phenacyl bromides through formation of host-guest complex during the course of reaction and thus facilitates the condensation (Figure 2).

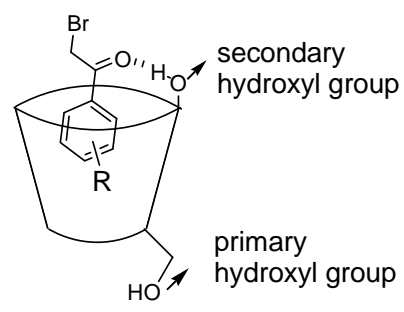

Figure 2. Host-guest complex of $\beta$-cyclodextrin and phenacyl bromide.

The evidence for the proposed mechanism could be deduced from ${ }^{1} \mathrm{H}$ NMR spectroscopy of $\beta$-CD and freeze-dried reaction mixture of $\beta$-CD-2-bromo-1-phenylethanone complex with 2aminopyrdine. Binding of phenacyl bromide into the cyclodextrin cavity was confirmed by the significant changes in the chemical shift of H3, H6 and H5 protons (located inside the cavity), whereas only little changes in the chemical shifts of $\mathrm{H} 2$ and $\mathrm{H} 4$ protons (located outside the cavity) of $\beta$-cyclodextrin were observed (Figure 3 ). This observation was consistent with inclusion complex formation between $\beta$-CD and phenacyl bromide. The structures of all the synthesized fused imidazoles were confirmed by spectroscopic data. The physical and spectroscopic data of the reported compounds were comparable with literature. 
Thus, we have developed an operationally simple and novel route for the preparation of bridgehead nitrogen heterocycles in water. The low cost, ease of handling and non-toxicity of $\beta$ cyclodextrin, makes the method employed in the present report an environment friendly.

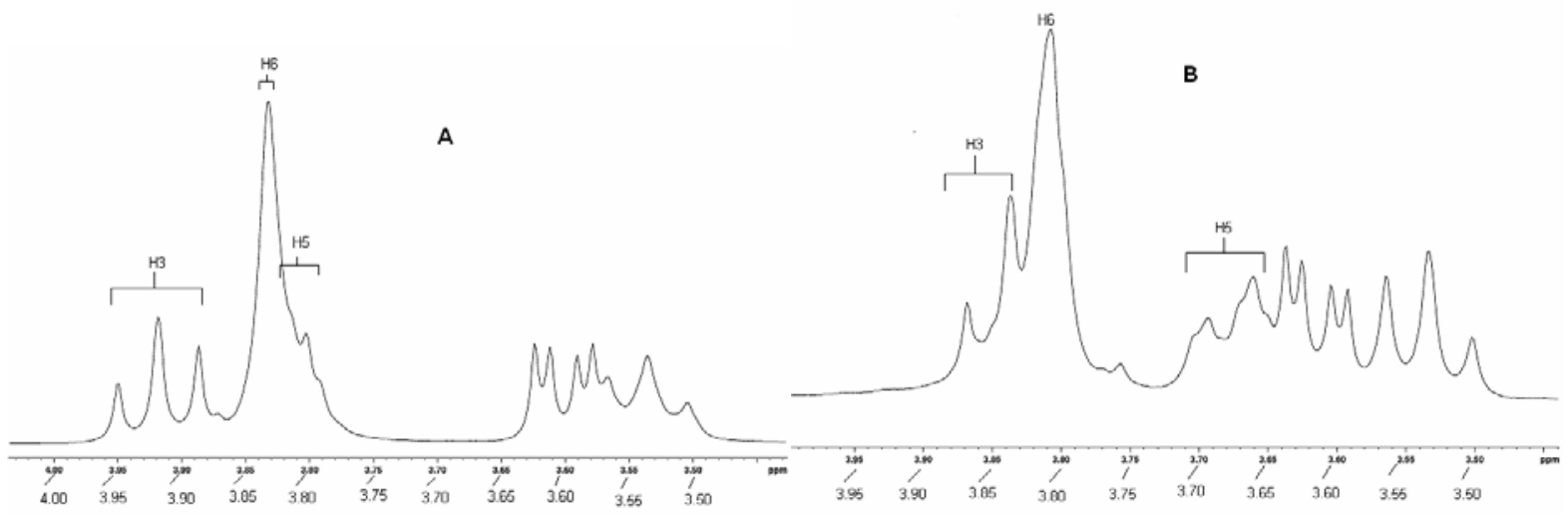

Figure 3. ${ }^{1} \mathrm{H}$ NMR spectra $\left(\mathrm{D}_{2} \mathrm{O}, 300 \mathrm{MHz}\right)$ of: $(\mathrm{A})$ : $\beta$-CD; $(\mathrm{B})$ : a freeze-dried reaction mixture of $\beta$-CD-phenacyl bromide complex with 2-aminopyridine after $15 \mathrm{~min}$.

\section{Experimental Section}

General Procedures. Reaction progress was monitored by TLC aluminium sheets silica gel 60 $\mathrm{F}_{254 .}{ }^{1} \mathrm{H}$ NMR and ${ }^{13} \mathrm{C}$ NMR spectra were recorded on Bruker Supercon Magnet DPX-200 or DRX-300 spectrometers (operating at 200 and $300 \mathrm{MHz}$ respectively for $1 \mathrm{H} ; 50$ and $75 \mathrm{MHz}$ respectively for $13 \mathrm{C}$ ) using $\mathrm{CDCl}_{3}, \mathrm{DMSO}-d_{6}, \mathrm{CD}_{3} \mathrm{OD}$ or their mixture as solvent. Infrared spectra were recorded on a Perkin-Elmer FT-IR RXI spectrophotometer. Electrospray mass spectra (ES-MS) were recorded on a Micromass Quattro II triple quadruple mass spectrometer. High-resolution electron impact mass spectra (HREIMS) were obtained on JEOL MS route $600 \mathrm{H}$ instrument. Elemental analysis were performed on Vario EL-III C H N S analyzer. Column chromatography was performed over Merck silica gel (particle size: 60-120 Mesh) procured from Qualigens (India), and flash silica gel (particle size: 230-400 Mesh). Commercially available grades of organic solvents of adequate purity are used in many reactions.

\section{General reaction conditions}

$\beta$-Cyclodextrin ( $1 \mathrm{mmol}$ ) was dissolved in $10 \mathrm{~mL}$ distilled water by heating at $50-55{ }^{\circ} \mathrm{C}$, to it was added dropwise, phenacyl bromide $(1 \mathrm{mmol})$ dissolved in acetone $(1 \mathrm{~mL})$. After five minutes of continuous stirring at same temperature, amine $(1 \mathrm{mmol})$ in acetone $(1 \mathrm{~mL})$ was added and the mixture was stirred at same temperature. After completion of the reaction, the crude products were isolated by extracting with ethyl acetate $(5 \mathrm{~mL} \times 3)$. The organic phase were separated and 
washed with brine, dried over sodium sulphate and evaporated under vacuum. The product was further purified by column chromatography using $2 \%$ methanol/chloroform.

2-(4-Chlorophenyl)-imidazo[1,2-a]pyridine (5a). ${ }^{1} \mathrm{H} \mathrm{NMR}\left(300 \mathrm{MHz}, \mathrm{CDCl}_{3}\right)$ : $\delta$ 6.77-6.81 (m, $1 \mathrm{H}), 7.16-7.21(\mathrm{~m}, 1 \mathrm{H}), 7.40(\mathrm{~d}, 2 \mathrm{H}, J 8.5 \mathrm{~Hz}), 7.62(\mathrm{~d}, 1 \mathrm{H}, J 9.0 \mathrm{~Hz}), 7.84(\mathrm{~s}, 1 \mathrm{H}), 7.89$ (d, 2H, $J 8.5 \mathrm{~Hz}), 8.1(\mathrm{~d}, 1 \mathrm{H}, J 6.8 \mathrm{~Hz}) ;{ }^{13} \mathrm{C} \mathrm{NMR}\left(50 \mathrm{MHz}, \mathrm{CDCl}_{3}\right): \delta 108.63(\mathrm{CH}), 113.16(\mathrm{CH})$, $117.85(\mathrm{CH}), 125.55(\mathrm{CH}), 126.05(\mathrm{CH}), 127.71(\mathrm{CH}), 129.33(\mathrm{CH}), 132.46(\mathrm{C}), 134.21(\mathrm{C})$, $144.86(\mathrm{C}), 145.99(\mathrm{C})$; IR (KBr) cm $\mathrm{cm}^{-1}$ : 1634, 760, 670; ES-MS (m/z): 229 [M+H] $]^{+}$; HRMS-EI: found: 228.0450 , calculated: 228.0455.

2-(4-Bromophenyl)-imidazo[1,2-a $]$ pyridine (5b). ${ }^{1} \mathrm{H}$ NMR (300 MHz, $\left.\mathrm{CDCl}_{3}\right)$ : $\delta$ 6.48-6.52 (m, 1H), 6.65-6.69 (m, 2H), 7.35 (d, 1H, J9.0 Hz), 7.51 (d, 2H, J 8.3 Hz), 7.60 (d, 2H, 7.5 Hz), 7.86 $(\mathrm{s}, 1 \mathrm{H}), 8.13(\mathrm{~d}, 1 \mathrm{H}, J 6.3 \mathrm{~Hz}) ;{ }^{13} \mathrm{C} \mathrm{NMR}\left(75 \mathrm{MHz}, \mathrm{CDCl}_{3}+\mathrm{DMSO}-d_{6}\right): \delta 109.33,113.26$,

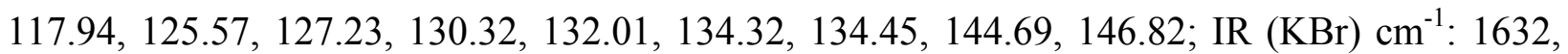
765, 506; ES-MS $(m / z): 273\left(\mathrm{Br}^{78}\right), 275\left(\mathrm{Br}^{80}\right)[\mathrm{M}+\mathrm{H}]^{+}$; HRMS-EI: found: 271.9937, calculated: 271.9950 .

2-(3,4-Dichlorophenyl)-imidazo[1,2-a]pyridine (5c). ${ }^{1} \mathrm{H}$ NMR (300 $\left.\mathrm{MHz}, \mathrm{CDCl}_{3}+\mathrm{DMSO}-d_{6}\right)$ : $\delta$ 6.96-7.00 (m, 1H), 7.34-7.41 (m, 1H), $7.53(\mathrm{~d}, 1 \mathrm{H}, J 8.4 \mathrm{~Hz}), 7.81-7.89(\mathrm{~m}, 2 \mathrm{H}), 8.09(\mathrm{~m}, 2 \mathrm{H})$, 8.34 (d, 1H, J 6.4 Hz); ${ }^{13} \mathrm{C}$ NMR (50 MHz, $\left.\mathrm{CDCl}_{3}+\mathrm{CD}_{3} \mathrm{OD}\right): \delta 110.02,113.99,114.68,124.88$, $126.95,127.30,129.09,129.84,130.58,132.69,133.70,143.91$; IR (KBr) cm${ }^{-1}: 1654,1463,760$, 673; ES-MS (m/z): 263 [M+H] ${ }^{+}$; HRMS-EI: found: 262.0065, calculated:. 262.0064.

2-(4-Methoxyphenyl)-imidazo[1,2-a]pyridine (5d). ${ }^{1} \mathrm{H}$ NMR (300 MHz, DMSO- $\left.d_{6}\right): \delta 3.82$ (s, $3 \mathrm{H}), 6.83-6.86(\mathrm{~m}, 1 \mathrm{H}), 7.02(\mathrm{~d}, 2 \mathrm{H}, J 8.2 \mathrm{~Hz}), 7.22-7.33(\mathrm{~m}, 1 \mathrm{H}), 7.56(\mathrm{~m}, 1 \mathrm{H}), 7.86(\mathrm{~d}, 2 \mathrm{H}, J$ $8.2 \mathrm{~Hz}), 8.30(\mathrm{~s}, 1 \mathrm{H}), 8.49-8.51(\mathrm{~m}, 1 \mathrm{H}) ;{ }^{13} \mathrm{C} \mathrm{NMR}\left(75 \mathrm{MHz}, \mathrm{CDCl}_{3}+\mathrm{DMSO}-d_{6}\right): \delta 55.80$, 108.32 , 113.32, 115.54, 116.81, 119.01, 126.20, 128.58, 132.81, 133.01, 145.86, 161.82; IR $(\mathrm{KBr}) \mathrm{cm}^{-1}: 1635,1247,760$; ES-MS $(\mathrm{m} / \mathrm{z}): 225[\mathrm{M}+\mathrm{H}]^{+}$; HRMS-EI: found: 224.0952, calculated:. 224.0950.

2-p-Tolylimidazo[1,2-a]pyridine (5e). ${ }^{1} \mathrm{H} \mathrm{NMR}\left(300 \mathrm{MHz}, \mathrm{CDCl}_{3}\right): \delta 2.39$ (s, 3H), 6.59-6.68 $(\mathrm{m}, 1 \mathrm{H}), 7.10-7.14(\mathrm{~m}, 1 \mathrm{H}), 7.23(\mathrm{~d}, 2 \mathrm{H}, J 7.9 \mathrm{~Hz}), 7.60-7.62(\mathrm{~m}, 1 \mathrm{H}), 7.80(\mathrm{~s}, 1 \mathrm{H}), 7.85(\mathrm{~d}, 2 \mathrm{H}$, $J 8.0 \mathrm{~Hz}), 8.06(\mathrm{~d}, 1 \mathrm{H}, J 6.8 \mathrm{~Hz}) ;{ }^{13} \mathrm{C} \mathrm{NMR}\left(75 \mathrm{MHz}, \mathrm{CDCl}_{3}+\mathrm{DMSO}-d_{6}\right): \delta 21.41,108.35$, 113.41, 117.82, 125.92, 126.31, 129.32, 130.21, 132.32, 134.29, 144.21; IR (KBr) cm $\mathrm{cm}^{-1}$ 1634, 765; ES-MS (m/z): 209 [M+H] $]^{+}$HRMS-EI: found: 208.1005, calculated: 208.1001.

2-Phenylimidazo[1,2-a]pyridine (5f). ${ }^{1} \mathrm{H}$ NMR (300 MHz, DMSO- $\left.d_{6}\right): \delta$ 6.85-6.91 (m, $\left.1 \mathrm{H}\right)$, 7.23-7.25 (m, 1H), 7.30-7.34 (m, 1H), 7.43-7.46 (m, 2H), 7.59 (d, 1H, J 6.9 Hz), 7.97-7.80 (m, $2 \mathrm{H}), 8.42(\mathrm{~s}, 1 \mathrm{H}), 8.52-8.54(\mathrm{~m}, 1 \mathrm{H}) ;{ }^{13} \mathrm{C}$ NMR $\left(50 \mathrm{MHz}, \mathrm{DMSO}-d_{6}\right): \delta 108.31,113.40,116.79$, $125.69,126.73,127.21,127.92,130.01,133.10,143.92$; IR $(\mathrm{KBr}) \mathrm{cm}^{-1}: 1634,762$; ES-MS $(\mathrm{m} / \mathrm{z}): 195[\mathrm{M}+\mathrm{H}]^{+}$; HRMS-EI: found: 194.0839, calculated: 194.0843.

6-Chloro-2-(3,4-dichlorophenyl)-imidazo[1,2-a]pyridine (5g). ${ }^{1} \mathrm{H}$ NMR (300 $\left.\mathrm{MHz}, \mathrm{CD}_{3} \mathrm{OD}\right)$ : $\delta$ 7.76-7.84 (m, 2H), $7.91(\mathrm{~d}, 1 \mathrm{H}, J 9.6 \mathrm{~Hz}), 8.00(\mathrm{dd}, 1 \mathrm{H}, J 9.6,1.6 \mathrm{~Hz}), 8.12(\mathrm{~d}, 1 \mathrm{H}, J 1.2 \mathrm{~Hz})$, $8.60(\mathrm{~s}, 1 \mathrm{H}), 9.01(\mathrm{~m}, 1 \mathrm{H}) ;{ }^{13} \mathrm{C} \mathrm{NMR}\left(50 \mathrm{MHz}, \mathrm{CD}_{3} \mathrm{OD}\right) \delta 111.24,111.53,124.28,124.82$, $125.50,126.02,127.08,130.46,132.40,133.52,136.88,138.51$; IR (KBr) cm ${ }^{-1}: 1650,1215,766$, 671; ES-MS (m/z): 297 [M+H] $]^{+}$; HRMS-EI: found: 295.9670, calculated: 295.9674. 
6-Chloro-2-phenylimidazo[1,2-a]pyridine (5h). ${ }^{1} \mathrm{H}$ NMR (300 $\left.\mathrm{MHz}, \mathrm{CDCl}_{3}+\mathrm{DMSO}-d_{6}\right): \delta$ 7.29-7.37 (m, 2H), 7.50-7.53 (m, 2H), 7.65 (d, 1H, J 7.0 Hz), 7.96-7.99 (m, 2H), $8.41(\mathrm{~s}, 1 \mathrm{H})$, 8.82-8.83 (m, 1H); IR (KBr) $\mathrm{cm}^{-1}$ : 1632, 750; ES-MS $(\mathrm{m} / \mathrm{z}): 229$ [M+H] $]^{+}$; HRMS-EI: found: 228.0459, calculated: 228.0454 .

6-Methyl-2-p-tolylimidazo[1,2-a]pyridine (5i). ${ }^{1} \mathrm{H} \mathrm{NMR}\left(300 \mathrm{MHz}, \mathrm{CD}_{3} \mathrm{OD}\right): \delta 2.44(\mathrm{~s}, 3 \mathrm{H})$, 2.50 (s, 3H), 7.42 (d, 2H, J 8.2 Hz), 7.76 (d, 2H, J 8.3 Hz), 7.77-7.87 (m, 2H), 8.42 (s, 1H), 8.59 $(\mathrm{s}, 1 \mathrm{H}) ;{ }^{13} \mathrm{C} \mathrm{NMR}\left(50 \mathrm{MHz}, \mathrm{CD}_{3} \mathrm{OD}\right) \delta 16.97,20.44,110.54,111.09,123.65,126.28,126.89$, 128.64, 130.30, 136.58, 136.68, 139.46, 141.55; IR (KBr) cm ${ }^{-1}: 1216,761,671$; ES-MS $(\mathrm{m} / z)$ : $223[\mathrm{M}+\mathrm{H}]^{+}$; HRMS-EI: found: 222.1159, calculated: 222.1156; Anal. Calcd for $\mathrm{C}_{15} \mathrm{H}_{14} \mathrm{~N}_{2}$ : C, 81.06; H, 6.35; N, 12.61. Found: C, 81.13; H, 6.31; N, 12.53.

6-Chloro-2-(4-methoxyphenyl)-imidazo[1,2-a]pyridine (5j). ${ }^{1} \mathrm{H}$ NMR $\left(300 \mathrm{MHz}, \mathrm{CD}_{3} \mathrm{OD}+\right.$ DMSO-d d $_{\text {) }} \delta 3.04(\mathrm{~s}, 3 \mathrm{H}), 6.33(\mathrm{~d}, 2 \mathrm{H}, J 8.8 \mathrm{~Hz}), 7.07$ (d, 2H, J 8.8 Hz), $7.14(\mathrm{~m}, 2 \mathrm{H}), 7.81(\mathrm{~s}$, $1 \mathrm{H}), 8.35(\mathrm{~s}, 1 \mathrm{H}) ;{ }^{13} \mathrm{C}$ NMR $\left(50 \mathrm{MHz}, \mathrm{CD}_{3} \mathrm{OD}+\mathrm{DMSO}-d_{6}\right) \delta 56.04,110.92,113.52,114.77$, $115.54,119.03,124.53,127.48,128.57,129.50,133.92,137.47,139.43,161.88 ; \mathrm{IR}(\mathrm{KBr}) \mathrm{cm}^{-1}$ : 1649, 1216, 760, 671; ES-MS $(\mathrm{m} / \mathrm{z}): 259$ [M+H] ${ }^{+}$; HRMS-EI: found: 258.0560, calculated: 258.0557; Anal. Calcd for $\mathrm{C}_{14} \mathrm{H}_{11} \mathrm{ClN}_{2} \mathrm{O}$ : C, 65.00; H, 4.29; N, 10.83. Found: C, 65.09; H, 4.26; $\mathrm{N}, 10.81$.

2-(4-Bromophenyl)-imidazo[1,2-a]pyrimidine (7a). ${ }^{1} \mathrm{H}$ NMR $\left(300 \mathrm{MHz}, \mathrm{CDCl}_{3}\right)$ : $\delta$ 6.94-6.97 $(\mathrm{m}, 1 \mathrm{H}), 7.57(\mathrm{~d}, 2 \mathrm{H}, J 8.4 \mathrm{~Hz}), 7.84(\mathrm{~s}, 1 \mathrm{H}), 7.93(\mathrm{~d}, 2 \mathrm{H}, J 8.5 \mathrm{~Hz}), 8.51-8.53(\mathrm{~m}, 1 \mathrm{H}), 8.79$ (dd, $1 \mathrm{H}, J$ 6.9, $2.1 \mathrm{~Hz}) ;{ }^{13} \mathrm{C} \mathrm{NMR}\left(75 \mathrm{MHz}, \mathrm{CD}_{3} \mathrm{OD}\right) \delta 108.19,109.51,124.89,129.79,132.30$, 133.01, 133.95, 138.23, 149.68, 152.72; IR (KBr) cm${ }^{-1}: 1630,760,504$; ES-MS (m/z): 274 $\left(\mathrm{Br}^{78}\right), 276\left(\mathrm{Br}^{80}\right),[\mathrm{M}+\mathrm{H}]^{+}$; HRMS-EI: found: 272.9909, calculated: 272.9901.

2-Phenylimidazo[1,2-a]pyrimidine (7b). ${ }^{1} \mathrm{H}$ NMR (300 MHz, $\left.\mathrm{CDCl}_{3}+\mathrm{DMSO}_{6}\right)$ : $\delta$ 6.81-6.83 $(\mathrm{m}, 1 \mathrm{H}), 7.43-8.16(\mathrm{~m}, 5 \mathrm{H}), 7.80(\mathrm{~s}, 1 \mathrm{H}), 8.42-8.43(\mathrm{~m}, 1 \mathrm{H}), 8.52-8.53(\mathrm{~m}, 1 \mathrm{H}) ;{ }^{13} \mathrm{C}$ NMR $(75$ $\left.\mathrm{MHz}, \mathrm{CD}_{3} \mathrm{OD}\right) \delta 108.21,108.93,127.40,127.83,128.62,130.40,133.86,138.13,149.53$, 152.16; IR (KBr) cm ${ }^{-1}: 1687,1597,1076,765$; ES-MS $(m / z): 196[\mathrm{M}+\mathrm{H}]^{+}$; HRMS-EI: found: 195.0796, calculated: 195.0794 .

2-(3,4-Dimethoxyphenyl)-imidazo[1,2-a]pyrimidine (7c). ${ }^{1} \mathrm{H}$ NMR $\left(300 \mathrm{MHz}, \mathrm{CDCl}_{3}\right): \delta 3.85$ $(\mathrm{s}, 3 \mathrm{H}), 3.87(\mathrm{~s}, 3 \mathrm{H}), 7.10-7.13(\mathrm{~m}, 1 \mathrm{H}), 7.65-7.70(\mathrm{~m}, 3 \mathrm{H}), 8.54(\mathrm{~s}, 1 \mathrm{H}), 8.55-8.56(\mathrm{~m}, 1 \mathrm{H})$, 9.05-9.07 (m, 1H); ${ }^{13} \mathrm{C}$ NMR $\left(75 \mathrm{MHz}, \mathrm{CD}_{3} \mathrm{OD}\right) \delta 56.03,56.10,109.01,110.21,110.32,112.04$, $121.81,127.11,133.86,138.61,149.73,151.81$; IR $(\mathrm{KBr}) \mathrm{cm}^{-1}: 1642,1082,730,665$; ES-MS $(\mathrm{m} / \mathrm{z})$ : $256[\mathrm{M}+\mathrm{H}]^{+}$; HRMS-EI: found: 255.1009, calculated: 255.1006; Anal. Calcd for $\mathrm{C}_{14} \mathrm{H}_{13} \mathrm{~N}_{3} \mathrm{O}_{2}$ : C, 65.87; H, 5.12; N, 16.46. Found: C, 65.94; H, 5.10; N, 16.51.

2-(3,4-Dichlorophenyl)-imidazo[1,2-a]pyrimidine (7d). ${ }^{1} \mathrm{H}$ NMR (300 MHz, DMSO- $\left.d_{6}\right): \delta$ 6.89-6.91 (m, 1H), 7.63-7.65 (m, 3H), $8.06(\mathrm{~s}, 1 \mathrm{H}), 8.56-8.57(\mathrm{~m}, 1 \mathrm{H}), 8.66-8.68(\mathrm{~m}, 1 \mathrm{H}) ;{ }^{13} \mathrm{C}$ NMR (75 MHz, DMSO-d $) \delta 107.89,109.31,127.71,128.31,130.32,133.01,133.35,133.83$, 134.51, 138.14, 148.32, 151.83; IR (KBr) $\mathrm{cm}^{-1}: 1658,1082,731,670 ;$ ES-MS $(\mathrm{m} / \mathrm{z}): 264$ [M+H] $]^{+}$; HRMS-EI: found: 263.0018, calculated: 263.0016.

2-(4-Chlorophenyl)-imidazo[1,2-a]pyrimidine (7e). ${ }^{1} \mathrm{H}$ NMR $\left(300 \mathrm{MHz}, \mathrm{CDCl}_{3}\right): \delta$ 6.87-6.89 $(\mathrm{m}, 1 \mathrm{H}), 7.45-7.89(\mathrm{~m}, 4 \mathrm{H}), 7.89(\mathrm{~s}, 1 \mathrm{H}), 8.42-8.44(\mathrm{~m}, 1 \mathrm{H}), 8.53-8.55(\mathrm{~m}, 1 \mathrm{H}) ;{ }^{13} \mathrm{C}$ NMR $(75$ 
MHz, DMSO- $\left.d_{6}\right) \delta 108.31,109.53,127.36,129.81,131.17,133.89,134.20,139.61,148.31$, 152.01; IR (KBr) cm $\mathrm{cm}^{-1}: 1611,1076$ 735, 675; ES-MS $(\mathrm{m} / \mathrm{z}): 207$ [M+H] ; HRMS-EI: found: 206.1096, calculated: 206.1094; Anal. Calcd for $\mathrm{C}_{12} \mathrm{H}_{8} \mathrm{ClN}_{3}$ : C, 62.75; H, 3.51; N, 18.30. Found: C, 62.82; H, 3.47; N, 18.27.

2-(4-Chlorophenyl)-imidazo[1,2-a]pyrazine (9a). ${ }^{1} \mathrm{H}$ NMR (300 MHz, $\left.\mathrm{CDCl}_{3}\right)$ : $\delta$ 7.44-7.49 (m, 2H), 7.91-7.93 (m, 2H), 7.95-7.97 (m, 2H), 8.08-8.10 (m, 1H), $9.13(\mathrm{~s}, 1 \mathrm{H}) ;{ }^{13} \mathrm{C}$ NMR $(50 \mathrm{MHz}$, $\mathrm{CDCl}_{3}+$ DMSO- $\left.d_{6}\right) \delta 109.80,119.13,126.04,127.69,130.01,130.44,131.62,133.60,142.79$, 143.86; IR (KBr) $\mathrm{cm}^{-1}: 2350,1610,1215,761,672$; ES-MS $(\mathrm{m} / \mathrm{z}): 230[\mathrm{M}+\mathrm{H}]^{+}$; HRMS-EI: found: 229.0408, calculated: 229.0406. Anal. Calcd for $\mathrm{C}_{12} \mathrm{H}_{8} \mathrm{ClN}_{3}: \mathrm{C}, 62.75 ; \mathrm{H}, 3.51 ; \mathrm{N}, 18.30$. Found: C, 62.84; H, 3.49; N, 18.27 .

2-Phenylimidazo[1,2-a]pyrazine (9b). ${ }^{1} \mathrm{H}$ NMR $\left(200 \mathrm{MHz}, \mathrm{CDCl}_{3}\right): \delta 7.39-7.53(\mathrm{~m}, 4 \mathrm{H}), 7.89$ $(\mathrm{d}, 1 \mathrm{H}, J 6.9 \mathrm{~Hz}), 7.96-8.01(\mathrm{~m}, 2 \mathrm{H}), 8.10(\mathrm{dd}, 1 \mathrm{H}, J 6.9,2.2 \mathrm{~Hz}), 9.12(\mathrm{~s}, 1 \mathrm{H}) ;{ }^{13} \mathrm{C}$ NMR $(50$ $\left.\mathrm{MHz}, \mathrm{CDCl}_{3}\right) \delta 109.70,119.11,126.88,128.78,129.35,129.95,130.45,133.07,133.59,143.91$; IR $(\mathrm{KBr}) \mathrm{cm}^{-1}: 2358,1595,1216,761,670$; ES-MS $(\mathrm{m} / \mathrm{z}): 196[\mathrm{M}+\mathrm{H}]^{+}$; HRMS-EI: found: 195.0798, calculated: 195.0796; Anal. Calcd for $\mathrm{C}_{12} \mathrm{H}_{9} \mathrm{~N}_{3}$ : C, 73.83; H, 4.64; N, 21.52. Found: C,73.89; H, 4.61; N, 21.55 .

2-(3,4-Dichlorophenyl)-imidazo[1,2-a]pyrazine (9c). ${ }^{1} \mathrm{H}$ NMR $\left(200 \mathrm{MHz}, \mathrm{CDCl}_{3}+\right.$ DMSO$\left.d_{6}\right): \delta 7.63-7.65(\mathrm{~m}, 3 \mathrm{H}), 7.93-7.96(\mathrm{~m}, 2 \mathrm{H}), 8.01-8.03(\mathrm{~m}, 1 \mathrm{H}), 8.90(\mathrm{~s}, 1 \mathrm{H}),{ }^{13} \mathrm{C} \mathrm{NMR}(50 \mathrm{MHz}$, $\left.\mathrm{CDCl}_{3}\right) \delta 109.69,119.01,127.76,128.29,129.89,130.10,130.43,130.53,131.50,133.58$, 133.71, 143.90, IR (KBr) $\mathrm{cm}^{-1}: 2281,1601,1197,758,672$; ES-MS $(\mathrm{m} / \mathrm{z}): 264[\mathrm{M}+\mathrm{H}]^{+}$; HRMSEI: found: 263.0017, calculated: 263.0016; Anal. Calcd for $\mathrm{C}_{12} \mathrm{H}_{7} \mathrm{Cl}_{2} \mathrm{~N}_{3}$ : C, 54.57; H, 2.67; N, 15.90. Found: C,54.65; H, 2.63; N, 15.84 .

2-(4-Bromophenyl)-imidazo[1,2-a]pyrazine (9d). ${ }^{1} \mathrm{H}$ NMR (300 MHz, $\left.\mathrm{CDCl}_{3}+\mathrm{DMSO}_{6} d_{6}\right): \delta$ 7.57 (d, 2H, $J 7.8 \mathrm{~Hz}), 7.80$ (d, 2H, $J .7 \mathrm{~Hz}), 7.94-8.00$ (m, 2H), 8.03-8.04 (m, 1H), $9.0(\mathrm{~s}, 1 \mathrm{H})$; ${ }^{13} \mathrm{C}$ NMR $\left(50 \mathrm{MHz}, \mathrm{CDCl}_{3}\right) \delta 108.90,118.91,122.11,127.58,128.79,130.43,130.62,130.72$ 133.61, 142.80, IR (KBr) cm ${ }^{-1}: 2300,1589,1203,750,671$; ES-MS $(\mathrm{m} / \mathrm{z}): 274\left(\mathrm{Br}^{78}\right), 276\left(\mathrm{Br}^{80}\right)$, [M+H] ${ }^{+}$; HRMS-EI: found: 272.9903, calculated: 272.9901; Anal. Calcd for $\mathrm{C}_{12} \mathrm{H}_{8} \mathrm{BrN}_{3}$ : C, 52.56; H, 2.94; N, 15.35. Found: C, 52.64; H, 2.91; N, 15.38 .

2-Phenyl-benzo[d]imidazo[2,1-b]thiazole (11a). ${ }^{1} \mathrm{H}$ NMR (300 MHz, $\left.\mathrm{CDCl}_{3}\right): \delta 6.45-6.97(\mathrm{~m}$, $6 \mathrm{H}), 7.57-7.58(\mathrm{~m}, 1 \mathrm{H}), 7.64-7.66(\mathrm{~m}, 1 \mathrm{H}), 7.88-7.89(\mathrm{~m}, 1 \mathrm{H}), 7.95(\mathrm{~s}, 1 \mathrm{H}) ;{ }^{13} \mathrm{C}$ NMR $(50 \mathrm{MHz}$, $\left.\mathrm{CDCl}_{3}\right) \delta 107.26,113.07,123.95,124.85,125.32,125.67,126.63,127.99,128.11,129.15$, 129.39, 140.12, 148.80; IR (KBr) $\mathrm{cm}^{-1}: 3400,2360,1592$; ES-MS $(\mathrm{m} / \mathrm{z}): 251[\mathrm{M}+\mathrm{H}]^{+}$; HRMSEI: found: 250.0567 , calculated: 250.0564 .

2-(4-Chlorophenyl)-benzo[d]imidazo[2,1-b]thiazole (11b). ${ }^{1} \mathrm{H}$ NMR $\left(300 \mathrm{MHz}, \mathrm{CDCl}_{3}\right): \delta$ 7.33-7.38 (m, 3H), 7.44-7.45 (m, 1H), 7.56-7.57 (m, 1H), 7.65-7.67 (m, 1H), 7.76-7.80 (m, $2 \mathrm{H})$, $7.91(\mathrm{~s}, 1 \mathrm{H}) ;{ }^{13} \mathrm{C}$ NMR $\left(50 \mathrm{MHz}, \mathrm{CDCl}_{3}\right) \delta 107.13,112.97,123.80,124.65,125.73,126.68$, $126.70,127.89,128.90,129.32,132.10,139.31,148.62$; IR (KBr) cm${ }^{-1}: 3431,2930,1590$; ESMS (m/z): 285 [M+H] ; HRMS-EI: found: 284.0178, calculated: 284.0176.

2-(4-Methoxyphenyl)-benzo[d]imidazo[2,1-b]thiazole (11c). ${ }^{1} \mathrm{H}$ NMR $\left(300 \mathrm{MHz}, \mathrm{CDCl}_{3}\right): \delta$ 3.85 (s, 3H), 6.93-6.97 (m, 2H), 7.25-7.45 (m, 2H), 7.46-7.47 (m, 1H), 7.53-7.54 (m, 1H), 7.66- 
$7.81(\mathrm{~m}, 2 \mathrm{H}), 7.85(\mathrm{~s}, 1 \mathrm{H}) ;{ }^{13} \mathrm{C} \mathrm{NMR}\left(50 \mathrm{MHz}, \mathrm{CDCl}_{3}\right) \delta 55.83,107.36,113.13,113.91,114.12$, 122.98, 123.83, 125.32, 128.55, 129.31, 139.11, 152.41, 160.43; IR (KBr) cm${ }^{-1}: 3401,2930$, 1598, 1487; ES-MS $(\mathrm{m} / \mathrm{z}): 281[\mathrm{M}+\mathrm{H}]^{+}$; HRMS-EI: found: 280.0680, calculated: 280.0670; Anal. Calcd for $\mathrm{C}_{16} \mathrm{H}_{12} \mathrm{~N}_{2} \mathrm{OS}$ : C, 68.55; H, 4.31; N, 9.99. Found: C, 68.57; H, 4.35; N, 9.94.

2-Benzofuran-2-yl-imidazo[1,2-a $]$ pyridine (12a). ${ }^{1} \mathrm{H} \mathrm{NMR}\left(300 \mathrm{MHz}, \mathrm{CDCl}_{3}\right)$ : $\delta$ 6.79-6.84 (m, 1H), 7.19-7.32 (m, 4H), 7.51-7.54 (m, 1H), 7.61-7.64 ( m, 2H), $7.99(\mathrm{~s}, 1 \mathrm{H}), 8.13-8.16(\mathrm{~m}, 1 \mathrm{H})$; ${ }^{13} \mathrm{C}$ NMR $\left(50 \mathrm{MHz}, \mathrm{CDCl}_{3}\right) \delta 103.41,109.99,111.53,113.28,118.02,121.61,123.40,124.82$, 125.83, 126.20, 129.44, 137.87, 146.29, 151.78, 155.31; IR (KBr) cm ${ }^{-1}: 2358,1654,1030,770$, 665; ES-MS (m/z): 235 [M+H] ${ }^{+}$; HRMS-EI: found: 234.0782, calculated: 234.0793; Anal. Calcd for $\mathrm{C}_{15} \mathrm{H}_{10} \mathrm{~N}_{2} \mathrm{O}$ : C,76.92; H, 4.31; N, 11.96. Found: C,76.96; H, 4.29; N, 11.91.

2-Benzofuran-2-yl-imidazo[1,2-a]pyrimidine (12b). ${ }^{1} \mathrm{H}$ NMR $\left(300 \mathrm{MHz}, \mathrm{CDCl}_{3}\right): \delta 6.90(\mathrm{dd}$, $1 \mathrm{H}, J 6.8,4.1 \mathrm{~Hz}), 7.23-7.28(\mathrm{~m}, 1 \mathrm{H}), 7.29-7.34(\mathrm{~m}, 1 \mathrm{H}), 7.43(\mathrm{~d}, 1 \mathrm{H}, J 0.5 \mathrm{~Hz}$, long range coupling), 7.52 (dd, 1H, J 7.9, $0.7 \mathrm{~Hz}), 7.65$ (dd, 1H, J 7.1, $1.5 \mathrm{~Hz}), 7.95$ (s, 1H), 8.46 (dd, 1H, $J$ 6.75, $2.0 \mathrm{~Hz}), 8.59$ (dd, $1 \mathrm{H}, J 4.0,2.0 \mathrm{~Hz}) ;{ }^{13} \mathrm{C} \mathrm{NMR}\left(75 \mathrm{MHz}, \mathrm{CDCl}_{3}+\mathrm{DMSO}_{-}\right)_{6} \delta 104.16$, 109.36, 109.83, 111.54, 121.90, 123.85, 125.43, 128.86, 135.89, 137.15, 148.65, 151.33, 152.10, 154.75; IR (KBr) $\mathrm{cm}^{-1}: 2362,1653,1028,771,668$; ES-MS $(\mathrm{m} / \mathrm{z}): 236[\mathrm{M}+\mathrm{H}]^{+}$; HRMS-EI: found: 235.0747, calculated: 235.0746; Anal. Calcd for $\mathrm{C}_{14} \mathrm{H}_{9} \mathrm{~N}_{3} \mathrm{O}: \mathrm{C}, 71.47 ; \mathrm{H}, 3.85 ; \mathrm{N}, 17.86$. Found: C, 71.50; H, 3.80; N, 17.79 .

\section{Acknowledgements}

S.K is thankful to the University Grants Commission, New Delhi, for financial support which is gratefully acknowledged and the Sophisticated Analytical Instrument Facility, CDRI, Lucknow for providing spectroscopic data.

\section{References}

1. Almirante, L.; Mugnaini, A.; De Toma, N.; Gamba, A. J. Med. Chem. 1970, 13, 1048.

2. Starrett, J. E.; Montzka, T. A.; Croswell, A. R.; Cavanagh, R. L. J. Med. Chem. 1989, 32, 2204.

3. Clements-Jewery, S.; Danswan, G.; Gardner, C. R.; Matharu, S. S.; Murdoch, R.; Tully, W. R.; Westwood, R. J. Med. Chem. 1988, 31, 1220.

4. Yamanaka, M.; Suda, S.; Kabasawa, Y.; Kawamura, T.; Ogawa, T.; Sawada, K.; Ohhara, H. Chem. Pharm. Bull. 1992, 40, 1486.

5. Kuwahara, M.; Kawano, Y.; Shimazu, H.; Yamamoto, H.; Ashida, Y.; Miyake, A. Chem. Pharm. Bull. 1995, 43, 1516.

6. Rival, Y.; Grassy, G.; Michel, G. Chem. Pharm. Bull. 1992, 40, 1170. 
7. Kaminski, J. J.; Bristol, J. A.; Puchalski, C.; Lovey, R. G.; Elliott, A. J.; Guzik, H.; Solomon, D. M.; Conn, D. J.; Domalski, M. S.; Wong, S. C.; Gold, E. H.; Long, J. F.; Chiu, P. J. S.; Steinberg, M.; Mc Phail, A. T. J. Med. Chem. 1985, 28, 876.

8. Barlin, G. B.; Brown, I. L.; Golic', L.; Kaucic', V. Aust. J. Chem. 1982, 35, 423.

9. Warshakoon, N. C.; Wu, S.; Boyer, A.; Kawamoto, R.; Sheville, J.; Renock, S.; Xu, K.; Pokross, M.; Evdokimov, A. G.; Walter, R.; Mekel, M. Bioorg. Med. Chem. Lett. 2006, 16, 5598.

10. Katritzky, A. R.; Xu, Y.-J.; Tu, H. J. Org. Chem. 2003, 68, 4935 and references cited therein.

11. Kovalska, V. B.; Losytskyy, M. Yu.; Kryvorotenko, D.V.; Balanda, A. O.; Tokar, V. P.; Yarmoluk, S. M. Dyes and Pigments 2006, 68, 39.

12. Yasumase, T.; Arima, H.; Tomioka, K.; Yamada, T.; Murase, K. J. Med. Chem. 1986, 29 , 386. (b) Tomoda, H.; Hirano, T.; Saito, S.; Mutai, T.; Araki, K. Bull. Chem. Soc. Jpn. 1999, 72, 1327. (c) Raival, Y.; Grassy, G.; Taudou, A.; Calle, R. E. Eur. J. Med. Chem. 1991, 26, 13.

13. Ponnala, S.; Kumar, S.T.V.S.K.; Bhat, B. A.; Sahu, D. P. Syn. Commun., 2005, 35, 7, 901.

14. (a) Ermolat'ev, D. S.; Gimenez, V. N.; Babaev, E. V.; Van der Eycken, E. J. Comb. Chem. 2006, 8, 659. (b) DiMauro, E. F.; Kennedy, J. M. J. Org. Chem. 2007, 72, 1013.

15. Rousseau, A. L.; Matlaba, P.; Parkinson, C. J. Tet. Lett. 2007, 48, 4079.

16. Franke, H.; Geisler, J.; Hartfiel, U.; Franke, W.; Dorfmeister, G.; Ganzer, M.; Johahann, G.; Rees, R. Ger. offen. DE. 4120 108, 1991; Chem. Abstr. 1992, 118, 213075v.

17. (a) Breslow, R.; Dong, S. D. Chem. Rev. 1998, 98, 1997. (b) Krishnaveni, N. S.; Surendra, K.; Kumar, V. P.; Srinivas, B.; Reddy, C. S.; Rao, K. R. Tetrahedron Lett. 2005, 46, 4299. (c) Surendra, K.; Krishnaveni, N. S.; Kumar, V. P.; Sridhar, R.; Rao, K. R. Tetrahedron Lett. 2005, 46, 4581. (d) Narender, M.; Reddy, M. S.; Sridhar, R.; Nageswar, Y. V. D.; Rao, K. R. Tetrahedron Lett. 2005, 46, 5953. (e) Chan, W. K.; Yu, W.Y.; Che, C. M.; Wong, M. K. J. Org. Chem. 2003, 68, 6576.

18. (a) Artyomov, V. A.; Shestopalov, A. M.; Litvinov, V. P., Synthesis. 1996, 927. (b) Guerret, P.; Jacquier, R.; Maury, G., J. Heterocycl. Chem. 1971, 8, 643. (c) Hunter, D.; Neilson, D. G. J. Chem. Soc., Perkin Trans. 1. 1988, 1439.

19. (a) Buu-Hoi, Ng. P.; Jacquignon, P.; Xuong, Ng. D.; Lavit, D. J. Org. Chem. 1954, 19, 1370.

(b) Singh, S. P.; Naithani, R; Prakasm, O. Indian Chem. Soc. 1998, 72, 770. (c) Buu-Hoi, Ng. Ph.; Xuong, Ng. D., Suu, V. T. J. Chem. Soc. 1958, 2815. (d) Katritzky, A. R; Qui, G.; Long, Q. H.; He, H. Y.; Steel, P. J. J. Org. Chem. 2000, 65, 9201.

20. Yasumase, T.; Arima, H.; Tomioka, K.; Yamada, T.; Murase, K. J. Med. Chem. 1986, 29, 386. 